http://dx.doi.org/10.12775/szhf.2013.035

DARIUSZ PAKALSKI

\title{
Der Polytechnikumstudent Castorp und Aufgabe der Kultur ${ }^{*}$
}

Aber was können die dort für eine technische Hochschule haben? Bestimmt eine miserable, das sag ich dir, mein Lieber - eine miserable, denn was ist das schon für eine Schule, die gerade erst gegründet wurde?

Diese Worte richtet der Hamburger Konsul James Tienappel an seinen Neffen Hans Castorp, der 1904 die Absicht hegt, an der Danziger Königlich Technischen Hochschule zu studieren. Das Zitat stammt jedoch nicht, wie man vermuten könnte, aus dem berühmten Roman Thomas Manns Der Zauberberg, wo bekanntlich die Geschichte des siebenjährigen Aufenthaltes dieses jungen, angeblich tuberkulosekranken Hanseaten im schweizerischen Luftkurort Davos geschildert wird. Der Autor dieser Worte ist der polnische Schriftsteller Paweł Huelle, der genau ein Jahrhundert später, von dem Zauberberg Manns inspiriert, seinen Roman Castorp veröffentlichte ${ }^{1}$. Als gebürtiger Danziger wurde Huelle von einem Satz aus der Geschichte Castorps elektrisiert. Thomas Mann schreibt nämlich in dem retrospektiven Kapitel über seinen Helden folgendes:

Dieses Referat wurde gehalten auf der Internationalen Wissenschaftlichen Konferenz „An den Quellen der Geistes- und Kulturwissenschaften ÖKONOMIE-KULTUR-WERTE“ anlässlich des 150. Geburtstages Heinrich Rickerts (15. 17. Mai 2013) an der Technischen Hochschule in Gdańsk.

${ }^{1}$ P. Huelle, Castorp, Gdańsk 2004, zit. nach der deutschen tbers. v. R. Schmidgall, München 2005, s. 7-8. 
Damals hatte er vier Semester Studienzeit am Danziger Polytechnikum hinter sich und vier weitere, die er auf den Technischen Hochschulen von Braunschweig und Karlsruhe verbracht hatte... ${ }^{2}$

Durch diesen Satz angeregt, trat Huelle mit einer sensationellen literarischen Hypothese auf. Er behauptete, Thomas Mann habe ein Danziger Kapitel des Zauberbergs verfasst, wo er die Studienzeit seines Helden in Danzig beschrieb, verzichtete aber später darauf, es in den Roman einzugliedern. Wenn man sich an die Geschichte des Zauberberg-Manuskripts erinnert, das mit fast hundertprozentiger Sicherheit im zweiten Weltkrieg während der Bombenangriffe auf München in Flammen aufgegangen ist ${ }^{3}$, lässt sich dieser Hypothese nicht alle Wahrscheinlichkeit absprechen, obwohl die MannForscher bisher von ihr keine Kenntnis haben. Und sollte Thomas Mann solch ein Kapitel jedoch nicht verfasst haben - setzt Huelle fort - dann müsse man es doch tun, weil Castorps Aufenthalt in Danzig ein interessantes und aufschlussreiches Thema für sich bilden könnte ${ }^{4}$. Warum sollte ein Ereignis, dem der deutsche Schriftsteller in der endgültigen Fassung nur rund zehn Worte widmet, irgendwelche Inhalte beherbergen, die für breites literarisches Publikum von Bedeutung sein könnten? In dem oben zitierten Fragment von Huelle fährt Konsul Tienappel fort, indem er auf diese Frage eine rätselhafte Antwort zu geben scheint, wobei er auch aus „unerklärlichen Gründen“ die Stimme senkt:

Und außerdem sollte man Situationen meiden, in denen die mühsam erarbeiteten Formen im Chaos versinken könnten ${ }^{5}$.

Wie nun zu erwarten ist, übersteigt diese Aufforderung die Kräfte des jungen Hanseaten, die „mühsam erarbeiteten Formen“, und Konsul Tienappel hatte hier wohl die Formen der bürgerlichen Kultur im Sinn, werden zerrüttet. Der frisch gebackene Schiffbaustudent erlebt während dieser vier Danziger Semester Dinge, angesichts deren sein Vetter Joachim Ziemßen, ein disziplinierter Unteroffizier der preußischen Armee, hätte nur ausrufen können

\footnotetext{
2 T. Mann, Der Zauberberg, Gesammelte Werke in 13 Bänden, Bd. 3, Frankfurt/M 1990, s. 55.

${ }^{3}$ Vgl. T. Mann, Der Zauberberg, Kommentar von M. Neumann, Große kommentierte Frankfurter Ausgabe, Bd. 5.2, Frankfurt/M 2002, s. 49-50.

${ }^{4}$ P. Huelle, Inne historie, Gdańsk 1999, s. 24-27.

${ }^{5}$ P. Huelle, Castorp, s. 8.
} 
„Guter Gott, das ist gegen die Ordnung “6. Wie wir sehen werden, wiederholt in dieser Hinsicht Huelles Roman die wichtigsten Motive aus dem Werk der klassischen deutschen Literatur.

Wie bekannt hat Der Zauberberg Thomas Manns seinen Höhepunkt in dem folgenreichen Kapitel Walpurgisnacht, das mit der Liebesvereinigung Hans Castorps mit seiner bezaubernden Geliebten Clawdia Chauchat endet, das aber eher an anrüchige Feste anknüpft, zu denen Hexen aus ganz Deutschland angeflogen waren, um ihrem Fürsten zu dienen. Der Leser ist hier Zeuge einer Fastnacht, diese Fastnacht entartet jedoch zu einer wahren Orgie, wo der Zeugungskraft und der bloßen Sexualität gehuldigt wird. Die Insassen des Davoser Sanatoriums Berghof personifizieren in dieser Szene pure Naturkräfte, entblößt von den Grundformen des Lebens. Der unschuldige Anfang dieser Ereignisse soll uns nicht täuschen. Zunächst wird nur das Faschingstraßenleben geschildert, wo unter den Passanten Masken, Pierroten und Harlekine unterwegs waren, aber als man sich später schon "hochgestimmt " ${ }^{\text {"7 }}$ zur Abendmahlzeit fand, beobachten wir weitere Stufen der „Travestierung "8. Einer der männlichen Insassen hatte einen Damenkimono und einen falschen Zopf angelegt, es sind Damen in Herrentracht erschienen, „die Gesichter bärtig geschwärzt mit angekohltem Flaschenkork “", und umgekehrt Männer in Frauenbekleidung, unter denen zum Beispiel ein Student „ein pickliges Dekolletté“ ${ }^{\prime 10}$ zur Schau stellte. Frauen verwandeln sich in Männer, Männer in Frauen, ein konkretes Geschlecht spielt keine Rolle mehr. Auf die Bühne tritt in einem Zitat von Goethes Faust eine Gestalt der griechischen Mythologie, die Gefährtin der Fruchtbarkeitsgöttin Demeter Baubo, die dadurch bekannt ist, ihre nach Entführung der Tochter Persephone trauernde Herrin durch obszöne Gesten aufheitern zu wollen, zu denen das Entblößen des Geschlechtsorgans gehört. Thomas Mann lässt uns in symbolischer Weise sehen, wie bei der Huldigung der bloßen Sexualität individuelle Züge des einzelnen Menschen aufgehoben werden, und das Individuum zur bloßen Gattung reduziert wird, zu einem puren biologischen Drang vom bisexuellen Charakter, wo der Trieb nach beiden Seiten tätig ist.

An dieser Stelle kommt unvermeidbar die Lehre eines namhaften Philosophen in den Vordergrund, deren Hauptanliegen war es zu zeigen, wie die

\footnotetext{
6 Tamże, s. 137.

7 T. Mann, Der Zauberberg, s. 451.

8 Tamże.

9 Tamże, s. 454.

${ }^{10}$ Tamże.
} 
raum-zeitlichen Grenzen des Principium individuationis zerschlagen werden und das Individuum sich in dem vernunft- und formlosen Willen auflöst. Der Wille als zeitloses, metaphysisches, triebhaftes Sein, liegt hier der Welt als Vorstellung zu Grunde. Das ist offensichtlich die Lehre Arthur Schopenhauers und dieser Philosoph wurde geboren - wo? In Danzig. Somit kommen wir zur Antwort auf die Paweł Huelle quälende Frage: warum lässt Thomas Mann seinen Helden gerade in der Stadt an der Mottlau, dem Geburtsort Arthur Schopenhauers studieren. Dieser Umstand bildet ein vermummtes, getarntes, literarisches Denkmal, das mit dem oben zitierten Satz von zehn Worten dem geistigen Vater des Zauberbergs errichtet wird. Auf den Einfluss Schopenhauers auf Thomas Mann ist in der deutschen Literaturforschung oft genug hingewiesen worden ${ }^{11}$, noch 1938 in seinem Essay Schopenhauer erinnert der Schriftsteller an den Rausch, in den ihn der "Zaubertrank“ seiner Metaphysik in der Jugend versetzt hatte ${ }^{12}$.

Das Motiv der Orgie können wir natürlich auch in dem Danziger Roman Castorp nicht entbehren. Von einem Kommilitonen eingeladen, der ihm Dinge zu sehen verspricht, „die nicht für einfache Lutheraner oder Katholiken bestimmt sind" ${ }^{\text {"13 }}$, landet der in Karnevalsstimmung versetzte junge Polytechnikumstudent irgendwo in einem Haus im Hafenviertel, und verfolgt da Begebenheiten, deren Prinzip es war - wie wir lesen - „die Bedürfnisse offen, ohne jegliche Scham oder Verhüllung, zu befriedigen ${ }^{\text {"14 }}$. Die Motive Manns - Maskerade, Travestierung und Bisexualität - werden wiederholt. Kurz bevor Castorp diese ausschweifende Veranstaltung verlässt, erliegt er, von Opium betäubt, der allgemeinen Atmosphäre der Sinnlichkeit, indem er einen "langen, intensiven Kuss" mit „einer athletischen Hera" ${ }^{15}$ tauschte, auf deren Wange unter der dicken Puderschicht der Bartwuchs zu spüren war. Auch seiner Danziger Liebe, der Polin Wanda Pilecka, die genauso wie die

${ }^{11}$ Die wichtigsten Beiträge zu diesem Thema sind: B. Kristiansen, Thomas Manns Zauberberg und Schopenhauers Metaphysik, Bonn 1986; M. Dierks, Studien zu Mythos und Psychologie bei Thomas Mann. An seinem Nachlaß orientierte Untersuchungen zum „Tod in Venedig“, zum „Zauberberg" und zur Joseph-Tetralogie, Frankfurt/M 20032; W. Frizen, Zaubertrank der Metaphysik, Quellenkritische Überlegungen im Umkreis der Schopenhauer-Rezeption Thomas Manns, Frankfurt/M-Bern-Cirencester 1980; H. Lehnert, Thomas Mann. Fiktion. Mythos. Religion, Stuttgart 1965; H. Wysling, Schopenhauer-Leser Thomas Mann, Schopenhauer-Jahrbuch 64, 1983, s. 61-79.

${ }_{12}$ T. Mann, Schopenhauer, Gesammelte Werke in 13 Bänden, Bd. 9, s. 561.

13 Tamże, s. 162.

14 Tamże, s. 164.

15 Tamże, s. 167. 
Russin Clawdia Chauchat „hervorstehende Wangenknochen“16 und „graublaue oder blaugraue" Augen hatte ${ }^{17}$, und von der später niemand Kenntnis nahm, begegnet Castorp in dieser seltsamen Nacht.

Die Heimsuchung durch Liebe als einen blinden Trieb, der über das Leben des Einzelnen seine Macht ausübt, ist wie bekannt die Schlüsselerfahrung vieler Helden Thomas Manns. Sie lassen sich durch diesen Trieb - wie man zum Beispiel über Gustav von Aschenbach lesen kann - „am Narrenseile ${ }^{\text {“18 }}$ leiten, und sind unabwendbar auf seine Wirkung angewiesen. Auf dem Gebiet der Metaphysik Schopenhauers ist aber die Liebe nur eine maskierte Figur des blinden Willens, einer Kraft, die nie aufhört, auf eine mehr oder weniger tragische Art und Weise das menschliche Leben zu determinieren, indem sie - so Thomas Mann - den ,heulende(n) Triumph der unterdrückten Triebwelt ${ }^{“ 19}$ mit sich bringt. In diese literarische Parabel - „Triumph der unterdrückten Triebwelt“ - ist bei Thomas Mann ein nicht mehr literarischer Gegensatz gekleidet, dieser Triumph bedeutet nämlich „die Niederlage der Zivilisation“. Nach der Selbstbekenntnis des Schriftstellers bedient er sich dieser Parabel, um den „Zusammenbruch einer mühsam, aus Einsicht und Verzicht gewonnenen hochkultivierten Haltung ${ }^{“ 20}$ zu veranschaulichen.

Unzweifelhaft ist das nur eine, nur dunkle Seite der Metaphysik Schopenhauers, aber von der anderen Seite dieser Metaphysik lässt sich jedoch nur sagen, dass sie noch dunkler ist. In der Szene aus der Walpurgisnacht bei Thomas Mann, wo in der Hauptrolle das Freudsche Symbol Bleistift besetzt worden war, ist Hans Castorp „totenbleich“, die Partie seines Gesichtes unter den Augen sieht „ganz so bleifarben wie bei einer Leiche ${ }^{\text {“21 }}$ aus, und er blickt in das Gesicht seiner Geliebten „aus seinen bleifarbenen Augenhöhlen“"22 - wie ein Toter. Thomas Mann vervollständigt somit seine literarische Version der Schopenhauerschen Philosophie. Neben Liebesmetaphysik stützt sie sich auf einem zweiten Pfeiler der Metaphysik des Todes. Nach der Lehre des Philosophen sind Zeugen und Sterben zwei immanente Korrelate des Lebens, und machen so das Wesen der unsterblichen Natur aus. Empfängnis und Tod sind demnach keine Grenzpunkte, die die Dauer des menschlichen Lebens ein-

\footnotetext{
16 Tamże, s. 118.

17 Tamże, s. 134.

${ }_{18}$ T. Mann, Der Tod in Venedig, Gesammelte Werke in 13 Bänden, Bd. 8, s. 520.

${ }^{19}$ T. Mann, On Myself, Gesammelte Werke in 13 Bänden, Bd. 13, s. 136.

${ }^{20}$ Tamże.

${ }^{21}$ T. Mann, Der Zauberberg, s. 463.

${ }^{22}$ Tamże, s. 464.
} 
schließen, sondern nur Momente, wo die Form des Lebens wechselt. Als ein bestimmtes Individuum, als eine konkrete Verwirklichung des Willens zum Leben, gewinnt das Leben einerseits eine bestimmte Form, gekennzeichnet durch Zeit, Raum und Kausalität. Hier erschließt sich auch das Feld für die Tätigkeit des Intellekts. Andererseits hört dieses Individuum nie auf, zu der allumfassenden Grundlage der Natur zu gehören, die ununterbrochen stirbt und wieder auflebt. Die Schopenhauersche Formel des Lebens ist nicht kompliziert, das Leben ist „durch und durch nichts Anderes - wie wir in Die Welt als Wille und Vorstellung lesen - als ein steter Wechsel der Materie, unter dem festen Beharren der Form "23. Der Geschlechtsakt und der Sterbensakt sind faszinierende Momente der Formzersprengung.

Liebe und Tod sind aber Markenzeichen nicht nur für die Philosophie Schopenhauers, sie sind auch Knotenpunkte einer geistigen Strömung, die sich in der deutschen Kulturgeschichte sehr stark ausgeprägt hat - der Romantik. Thomas Mann bezeichnete die wichtigsten Werke der ersten Hälfte seines Lebens, wie Tonio Kröger, Der Tod in Venedig und Der Zauberberg, als par excellence romantische Konzeptionen ${ }^{24}$. Es handelt sich hier um einen besonderen Aspekt der Romantik, der in einem prägenden Werk des Dichters Novalis seinen Ausdruck gefunden hat. Seine Hymnen an die Nacht sind eine literarische Niederschrift der Erschütterung über den frühen Tod seiner Braut Sophie von Kühn. Liebesleid und die Sehnsucht nach Liebesvereinigung bedeuten hier nichts Anderes als Todessehnsucht. Thomas Mann bezeichnet diese Einstellung mit der Formel „Sympathie mit dem Tode“. „Sympathie mit dem Tode“ - schreibt er in einem Essay - „fasst [...] gewiss das Wesen der Romantik nicht genau, aber sie bestimmt ihr Tiefstes und Höchstes" ${ }^{\text {"25. In } B e-}$ trachtungen eines Unpolitischen - einer riesenhaften Abhandlung, in der der Schriftsteller über seine geistige Haltung angesichts der europäischen Katastrophe des Ersten Weltkriegs Rechenschaft gibt - erscheint diese Formel im Kontext des Zauberbergs.

Vor dem Krieg - schreibt Thomas Mann - hatte ich einen kleinen Roman zu schreiben begonnen, eine Art von pädagogischer Geschichte, in der ein junger

\footnotetext{
${ }^{23}$ A. Schopenhauer, Die Welt als Wille und Vorstellung, Zürich 1988, Bd. 1, s. 364 . Zur Interpretation dieser Szene aus Walpurgisnacht vgl. D. Pakalski, Pytanie o ideę kultury w „Czarodziejskiej górze" Tomasza Manna, Toruń 2012, Kapitel Ciemny filozof Schopenhauer.

${ }^{24}$ T. Mann, Brief an Ernst Fischer vom 25.5.1926, in: Briefe III, Große kommentierte Frankfurter Ausgabe, Bd. 23, Frankfurt/M 2011.

${ }^{25}$ T. Mann, Von Deutscher Republik, Gesammelte Werke in 13 Bänden, Bd. 11, s. 849.
} 
Mensch, verschlagen an einen sittlich gefährlichen Aufenthaltsort, zwischen zwei gleichermaßen schnurrige Erzieher, Humanisten, Rhetor und Fortschrittsmann und einen etwas anrüchigen Mystiker, Reaktionär und Advokaten der Anti-Vernunft, - er bekam zu wählen, der gute Junge, zwischen den Mächten der Tugend und der Verführung, zwischen der Pflicht und dem Dienst des Lebens und der Faszination der Verwesung, für die er nicht unempfänglich war; und die Redewendung von der Sympathie mit dem Tode war ein thematischer Bestandteil der Komposition ${ }^{26}$.

In der endgültigen Fassung des Romans gewinnt die Wahl, vor der Castorp in diesem Fragment gestellt wurde, die Alternative zwischen Settembrini und Naptha, erst dann an Bedeutung, als seine Faszination durch den Tod schon geheilt ist. In dieser Phase sind die Disputationen beider Erzieher nur ein Nachklang, eine intellektuelle Bearbeitung des Konflikts, die der Leser genauso langweilig finden mag, wie ihn der eigentliche Konflikt am Anfang gefesselt hatte. Die ursprüngliche Form dieses Konflikts kommt woanders her, er wird von Madame Chauchat ausgelöst, es ist die Person der anziehenden Russin, mit der die Ideen der Liebe, der Schönheit und des Todes untrennbar verbunden sind. Der kränkliche Vertreter der bürgerlichen Kultur ist mit überwältigenden Mächten konfrontiert, die als Verführung, „als Trieb unserer Elemente zur Freiheit, zur Unform und zum Chaos erfasst ${ }^{\text {“27 }}$ werden. Das ist eben nichts Anderes als die oben erwähnte Situation, die zu vermeiden Onkel Tienappel seinem Zögling angeraten hatte. Sprechen wir in diesem Kontext über Romantik, dann bedeutet das so viel wie Irrationalität.

Noch eine Erfahrung Hans Castorps ist in dieser Hinsicht bedeutungsschwer. Wie bekannt betont Schopenhauer in seiner Metaphysik die Rolle der Musik. Für ihn bedeutet sie ein „Abbild des ganzen Willens“ oder ein „Abbild des Willens selbst ${ }^{\text {“28. }}$. Und tatsächlich, genauso wie die Dichtung hat auch die Musik dem Schicksal Castorps ihren Stempel aufgedrückt. Oder treffender gesagt ist es die Verbindung beider. Wilhelm Müller zählte seinerzeit zu den gefragtesten Dichtern Deutschlands. Sein Gedichtzyklus, heute unter dem Titel Winterreise bekannt, wurde von Franz Schubert vertont. Es entstand gleichsam ein Monolog in Liederform, der aus 24 Abschnitten besteht. Die Szenerie der Winterreise lässt sich leicht vermuten, enttäuschte Liebe treibt einen Unglücklichen in die froststarrende Winterwelt, wo ihm der Lebens-

\footnotetext{
${ }^{26}$ T. Mann, Betrachtungen eines Unpolitischen, tamże, Bd. 12, s. 424.

27 T. Mann, tamże, Bd. 11, s. 851.

${ }^{28}$ A. Schopenhauer, Die Welt als Wille und Vorstellung, Bd. 1, s. 341.
} 
wille immer mehr erlischt. Die Erstarrung der winterlichen Natur ist ein bekanntes Symbol des Todes, am Ende des Zyklus lauert die Selbstaufgabe und der Wille zur Flucht aus Raum und Zeit.

Im Roman Huelles sehen wir Castorp nach der abenteuerlichen Karnevalsnacht auf dem Danziger Fluss Mottlau schlendern. Der Fluss ist vereist, die Luft ist frostig, die Speicherinsel liegt in tiefem Schnee. Und plötzlich sieht Castorp „in absoluter Stille“ die Sonnenkugel sich in drei Sonnen teilen. Der polnische Schriftsteller wiederholt in dieser Szene ein Motiv aus dem Liederzyklus Schuberts. Lesen wir ein paar Zeilen aus dem Lied Die Nebensonnen:

Drei Sonnen sah ich am Himmel stehn,

Hab lange und fest sie angesehn;

und weiter:

Ach neulich hatt ich auch wohl drei:

Nun sind hinab die besten zwei.

Ging nur die dritt erst hinterdrein!

Im Dunkel wird mir wohler sein ${ }^{29}$.

Dieses „Im Dunkel wird mir wohler sein“ ist genau das, was für Castorp so anziehend war, und was er in Danzig nachempfindet: „Das einzige, was er jetzt wollte - lesen wir bei Huelle - war, sich mitten auf den gefrorenen Fluß legen und für immer einschlafen ${ }^{\text {“30. }}$.

Trotzdem hat er diese Versuchung überstanden. Mit „fast unmenschlicher Anstrengung" schaffte der schwächliche Junge es schließlich zum Kai in der Nähe der Grünen Brücke, wo wie jeden Morgen der schlichten Pflicht des Lebens nachgegangen wurde.

Im Thomas Manns Davoser Roman widersteht Castorp einer noch stärkeren Versuchung. Er reißt sich von der siebenjährigen Verzauberung los, in die ihn die dunklen Mächte des Schopenhauerischen Willens gebannt haben. Seine Metamorphose vollzieht sich im entscheidenden Kapitel Schnee. Als Erinnerung daran und als Abschied von der Welt der Verwesung singt er in der Abschlussszene des Buches ein Lied vor sich hin, und das ist Der Lindenbaum, ein anderes Lied aus Schuberts Winterreise:

Und seine Zweige rauschten,

Als riefen sie mir zu:

${ }^{29}$ Zitiert nach: www.projekt.gutenberg.de

${ }^{30}$ P. Huelle, Castorp, s. 168. 
»Komm her zu mir, Geselle,

Hier findst du deine Ruh!«

Keine Metamorphose des Geistes - schreibt Thomas Mann, und diese Worte beziehen sich wohl auf Castorp - ist uns besser vertraut als die, an deren Anfang die Sympathie mit dem Tode, an deren Ende der Entschluß zum Lebensdienste steht ${ }^{31}$.

Wenn man das System Schopenhauers und die Philosophie Heinrich Rikkerts, des Schirmherrn dieser Konferenz, in Vergleich zieht, findet man nur wenige Berührungspunkte. Der erste trägt eher einen biographischen Charakter. Rickert und Schopenhauer können sich desselben Geburtsortes rühmen, es ist wie bekannt die Stadt Danzig. Wir wissen auch, dass Rickerts Vater, ein einflussreicher Verleger der Danziger Zeitung, im Jahre 1897 die Anregung gab, in dieser abgelegenen, östlichen Provinzstadt ein Polytechnikum zu gründen, die Hochschule also, an der ein paar Jahre später der literarische Held Castorp sein Schiffbaustudium aufnahm. Und es ist die zweite Tatsache, die die beiden Philosophen in Zusammenhang bringt. Die Metamorphose, von der Thomas Mann schrieb, ist unzweifelhaft ein ethischer Akt, es ist ein Akt der Absage an die Haltung, sich gehenzulassen, seinen Neigungen nachzugehen, an die Haltung der moralischen Faulheit. Im Lichte Schopenhauers bedeutet dies einen Triumph des metaphysischen Willens, der im Grunde genommen einen Triumph der Biologie über ihr Produkt das Intellekt - mit sich bringt und die in der Kultur erarbeiteten Formen in ein Chaos verwandelt. Und eben Rickert war derjenige, der behauptete, als er die Lebensphilosophie kritisierte, dass das Leben „als bloßes Leben unter philosophischen Gesichtspunkten ein Chaos [bleibt] "32.

Im Vergleich zu Schopenhauer war Rickert ein Philosoph, der die Aufgabe des Lebens und die Aufgabe der Kultur besser definiert hat. Selbst wenn man die ethischen Postulate Schopenhauers in Betracht zieht, sein Programm der Weltentsagung, der Verneinung des Willens, seine ganze Philosophie des Kreuzes, dann bekommen wir nur eine Kehrseite der gleichen Medaille, das Kreuz bleibt eine Todesstelle, auch wenn man als Heiliger daran stirbt. In diesem Sinn beurteilte Mann die Philosophie Schopenhauers als lebenswidrig. Im Gegensatz dazu steht Rickert - wie Thomas Mann sagen würde im Dienste des Lebens. Seine Philosophie ist ein Versuch dem Leben Werte

${ }^{31}$ T. Mann, Von Deutscher Republik, s. 851.

${ }^{32}$ H. Rickert, Die Philosophie des Lebens, Tübingen 1920, s. 181. 
$\mathrm{zu}$ verleihen und dadurch seine geistigen Formen zu erarbeiten, was Rickert im engeren Sinne als das Sollen eines jeden Menschen, und im breiteren als Aufgabe der Kultur erfasst. Im Lichte vom Gedanken Rickerts ist Hans Castorp ein literarischer Held, der, selbst wenn er dieser Aufgabe nicht bewusst nachstrebt und sie nicht verwirklicht, durch seine Wahl auf diese Aufgabe zumindest hinweist. Seine Metamorphose ist ein literarisches Beispiel für die geschichtsphilosophische Verwandlung des "transzendenten Seins“ in das „transzendente Sollen“.

Ich möchte meinen kurzen Beitrag, dessen Thema eigentlich mehr Literatur als Philosophie war, auch mit einem literarischen, d.h. fiktiven Bild abschließen. Die Handlung des Romans Castorp von Paweł Huelle wechselt im zweiten Teil nach Zoppot.

Für die Geschichte Zoppots dürfte es kaum von Bedeutung gewesen sein - lesen wir darin - daß der Student Hans Castorp schon im ersten Jahr nach der Eröffnung des Warmbades Patient dieser Einrichtung wurde ${ }^{33}$.

In diesem Seebad, ein paar Kilometer von Danzig entfernt, konnte man zu dieser Zeit nach Angabe des Verfassers in 58 Wannen Rheumatismus, chronische Ausschläge, Leberschwellungen, Katarrh, Arthrose, Warzen, Herzflattern, Heiserkeit, Hämorrhoiden, Impotenz, Epilepsie, Geisteskrankheiten sowie alle Arten von Nervenschwächen behandeln, welch letztere Unpässlichkeit Hans Castorp nach seinen ausschweifenden und die Gesundheit überstrapazierenden Erlebnissen der letzten Monate zuteilwurde. Als er erfahren hat, dass die geheimnisvolle Polin für den Sommer 1905 ein Zimmer in der Pension Miramare gebucht hat, tut er dasselbe. Die Phantasie lässt uns an dieser Stelle ein imaginäres Bild aufkommen. Aus der Biographie Thomas Manns wissen wir, dass er die zweite Augusthälfte des gleichen Sommers mit seiner frisch geheirateten Frau Katia auch in Zoppot verbracht hatte ${ }^{34}$. Man kann sich gut eine Begegnung vorstellen, irgendwo im Park, auf der Terrasse des Kurhauses, auf dem Seesteg oder während eines abendlichen Strandspaziergangs, eine Begegnung unseres literarischen Helden mit seinem zukünftigen Autor. Hätten sie Grüße gewechselt? Es ist nicht ausgeschlossen, dass auch der Philosoph Heinrich Rickert in diesem Sommer sein Elternhaus in der zukünftigen Rickertstrasse in Zoppot besucht hatte. Und so sehe ich die

\footnotetext{
${ }^{33}$ P. Huelle, Castorp, s. 171.

${ }^{34}$ G. Heine, P. Sommer, Thomas Mann Chronik, Frankfurt/M 2004, s. 39-40.
} 
drei, vielleicht auf einer Bank sitzend, Castorp ein Band Schopenhauer in der Hand sich in seine Lehre vertiefend, „der er die nächsten zwei Semester seines Nachdenkens - wie wir bei Huelle lesen - am hiesigen, provinziellen, aber dennoch nicht zweitrangigen Polytechnikum widmete ${ }^{\text {"35. }}$.

\section{Abstract \\ The Polytechnic Student Castorp and the Role of Culture}

Keywords: Thomas Mann, Arthur Schopenhauer, metaphysics of will, culture, ethics

In his Magic Mountain Thomas Mann has placed a sentence that has its own history. He remarked that Hans Castorp spent four terms studying ship building on Gdańsk Polytechnic. Polish writer, Paweł Huelle (what is remarkable, born in Gdańsk), fascinated by this sentence, wrote a novel describing the Gdańsk episode of the character of Magic Mountain; the title of the novel is Castorp. The article focuses the essential motifs of both novels in the perspective of the question about culture.

As is widely known, one of the most important inspirations of Mann's writings is the philosophy of Arthur Schopenhauer. His metaphysics of will, the play of the powers of generating and destroying, is seen by Mann as a project very attractive for artistic purposes. Nevertheless, as Mann sees it, the project whose the ultimate result is melting individual in the shapeless world of metaphysical will is hostile towards life and endangers the forms of culture. Castorp, a literary character, subjugated by dark and blind powers of will, is an example of man who acknowledges the fact, but opts for life. He undertakes an attempt to restrain the powers and to give them civilized and more agreeable form, which is an attempt to struggle for the value of life, which in Mann's interpretation has a moral character. According to the philosophy of Heinrich Rickert Castorp, the literary character, is a man who strives for improving his own nature and sees it as an activity that is to be fulfilled in the sphere of culture. He wants to subject his conduct and activity to norms and settled values, treating this attitude as the ethical imperative. He strives for elaborating timeless values as a counterbalance for the metaphysical being of will. In a wider context it depicts the process in which the transcendent being is replaced by "transcendent obligation".

${ }^{35}$ P. Huelle, Castorp, s. 247. 